\title{
Autophagy mediated by endoplasmic reticulum stress enhances the caffeine-induced apoptosis of hepatic stellate cells
}

\author{
YONGJIAN LI ${ }^{1,2^{*}}$, YUNYANG CHEN ${ }^{1-3^{*}}$, HAIYAN HUANG ${ }^{1}$, MINMIN SHI $^{2}$, \\ WEIPING YANG $^{1,2}$, JIE KUANG $^{1,2}$ and JIQI YAN ${ }^{1,2}$ \\ ${ }^{1}$ Department of General Surgery and ${ }^{2}$ Shanghai Institute of Digestive Surgery, Ruijin Hospital, \\ Shanghai Jiao Tong University School of Medicine, Shanghai 200025; ${ }^{3}$ Department of Hepatobiliary Surgery, \\ Jiangmen Central Hospital, Jiangmen, Guangdong 529030, P.R. China
}

Received September 11, 2016; Accepted September 18, 2017

DOI: $10.3892 / \mathrm{ijmm} .2017 .3145$

\begin{abstract}
Caffeine has been identified to have beneficial effects against chronic liver diseases, particularly liver fibrosis. Many studies have reported that caffeine ameliorates liver fibrosis by directly inducing hepatic stellate cell (HSC) apoptosis; however, the molecular mechanisms involved in this process remain unclear. The presents study aimed to detect the underlying mechanisms by which caffeine mediates HSC apoptosis and eliminates activated HSCs. For this purpose, the LX-2 cell line was applied in this study and the cells were exposed to various concentrations of caffeine for the indicated times. The effects of caffeine on cell viability and apoptosis were assessed by Cell Counting Kit- 8 assay and flow cytometry, respectively. Autophagy and endoplasmic reticulum (ER) stress were explored by morphological assessment and western blotting. In the present study, caffeine was found to inhibit the viability and increase the apoptosis of the LX-2 cells in dose- and timedependent manners. ER stress was stimulated by caffeine as demonstrated by increased levels of GRP78/BiP, CHOP and inositol-requiring enzyme 1 (IRE1)- $\alpha$ as well as many enlarged ERs detected by electron microscopy. Caffeine induced autophagy as shown by increased p62 and LC3II accumulation and the number of GFP/RFP-LC3 puncta and autophagosomes/ autolysosomes. Moreover, IRE1- $\alpha$ knockdown decreased the level of autophagic flux, and inhibition of autophagy protected LX-2 cells from apoptotic death. In conclusion, our study showed that the caffeine-enhanced autophagic flux in HSCs
\end{abstract}

Correspondence to: Dr Jiqi Yan or Dr Jie Kuang, Department of General Surgery, Ruijin Hospital, Shanghai Jiao Tong University School of Medicine, 197 Ruijin Er Road, Shanghai 200025, P.R. China

E-mail: yanjiqi@sohu.com

E-mail: kuangjie823@msn.com

${ }^{*}$ Contributed equally

Key words: caffeine, endoplasmic reticulum stress, autophagy, inositol-requiring enzyme- $1 \alpha$, liver fibrosis was stimulated by ER stress via the IRE1- $\alpha$ signaling pathway, which further weakened HSC viability via the induction of apoptosis. These findings provide new insight into the mechanism of caffeine's anti-fibrotic effects.

\section{Introduction}

Liver cirrhosis is a consequence of diverse mechanisms of liver injury that lead to a wound-healing reponse characterized by necroinflammation and fibrogenesis $(1,2)$. Hepatic stellate cells (HSCs) play a critical role in the transition from chronic liver injury to cirrhosis (3). Upon liver injury, HSCs transdifferentiate from quiescent cells to activated, myofibroblast-like cells, resulting in proliferation, excessive production and secretion of extracelluar matrix $(4,5)$. Thus, the suppression of activated HSCs has been recognized as an effective pathway through which to attenuate liver fibrosis.

Autophagy is a degradation pathway by which the cell self-digests its own components by forming autophagosomes and autolysosomes (4). This self-digestion process occurs in all types of cells at a low basal level, serving a homeostatic function to recycle proteins and organelles. When the cell is going to rid itself of starvation, damaged organelles and superfluous or misfolded proteins, autophagy is rapidly upregulated (6). Moreover, recent studies have revealed that autophagy could be instigated by the upregulation of endoplasmic reticulum (ER) stress $(7,8)$.

ER is a type of flattened sac or tube-like organelle in eukaryotic cells, serving many essential functions, such as correctly folding newly made protein molecules and a $\mathrm{Ca}^{2+}$ reservoir $(9,10)$. Increased protein synthesis load and accumulation of unfolded or misfolded proteins in ER may disturb ER homeostasis, thereby imposing ER stress and the unfolded protein response (UPR) $(11,12)$. The UPR, initially, compensates for the damage caused by ER stress and maintains celluar homeostasis via enhancing the protein-folding ability of the ER and facilitating proteasomal degradation of unfolded or misfolded proteins. However, if ER stress is excessive or prolonged that transcends the protective ability of UPR, the high-intensitive UPR eventually results in cell apoptosis (13-15).

Caffeine, the major compound in coffee, has been identified to have a potential beneficial effect against 
liver fibrosis $(16,17)$. Recent findings have revealed that caffeine-mediated attenuation of liver fibrosis is probably associated with HSC apoptosis (18). Another study found that caffeine induced apoptosis of SH-SY5Y, PC12D and HeLa cells by stimulating autophagic flux by inhibiting the PI3K/ AKT/mTOR pathway (19). However, little is known concerning the mechanism by which caffeine regulates the apoptosis of HSCs.

Here, we report that caffeine-enhanced autophagic flux in HSCs was stimulated by ER stress through the IRE1- $\alpha$ pathway. Furthermore, enhanced autophagy attenuated the expression of $\alpha$-SMA by instigating HSC apoptosis. Therefore, our results provide new insight into the mechanism of caffeine's anti-fibrotic effects.

\section{Materials and methods}

Cell culture and compounds. LX-2 cells, a type of immortalized human HSC line, was purchased from the Cell Center of Xiangya School of Medicine, Central South University (Hunan, China), cultured in Dulbecco's modified Eagle's medium (DMEM) and supplemented with $10 \%$ fetal bovine serum (FBS) (both from Gibco, Grand Island, NY, USA), $100 \mathrm{IU} / \mathrm{ml}$ penicillin and $100 \mu \mathrm{g} / \mathrm{ml}$ streptomycin at $37^{\circ} \mathrm{C}$ in $5 \% \mathrm{CO}_{2}$. To measure the autophagic flux of LX-2 cells after being treated with caffeine, LX-2 cells were transfected with RFR-GFR-hLC3 lentivirus as previously described elsewhere (20). Caffeine (Sigma-Aldrich, St. Louis, MO, USA) was dissolved and diluted with DMEM to the desired concentrations which we selected according to other articles $(16,18,19)$. 3-Methyladenine (MA) (Sigma-Aldrich) was dissolved in phosphate-buffered saline (PBS) and diluted with DMEM to the concentration of $5 \mathrm{mM}$.

Western blotting. LX-2 cells were lysed in RIPA (Solarbio, Beijing, China) supplemented with protease inhibitor cocktail (Sigma-Aldrich) and the protein samples were centrifuged for $30 \mathrm{~min}$ at $10,000 \mathrm{x} \mathrm{g}\left(4^{\circ} \mathrm{C}\right)$. The supernatants were collected and the BCA protein assay kit (Pierce, Rockford, IL, USA) was applied to detect the protein concentration. The protein samples were separated by $12.5 \%$ SDS-PAGE and subsequently electrotransferred onto PVDF membranes. The membranes were blocked with $5 \%$ defatted milk for 1.5 to $2 \mathrm{~h}$ and incubated with the primary antibody overnight at $4^{\circ} \mathrm{C}$. The primary antibodies were LC3 (4108; Cell Signaling Technology, Inc., Danvers, MA, USA), SQSTM1 (ab109012; Abcam, Cambridge, UK), $\alpha$-SMA (A7249; ABclonal, Cambridge, MA, USA), Gpr78 (ab21685) and IRE1- $\alpha$ (ab124945) (both from Abcam) and CHOP (2895; Cell Signaling Technology, Inc., Danvers, MA, USA). Afterwards, the membranes were incubated with the HRP-conjugated secondary antibodies (SA00001-1 and SA00001-2; Proteintech Group, Inc., Rosemont, IL, USA) at room temperature for $1.5 \mathrm{~h}$. Finally the results were visualized by enhanced chemiluminescence.

Flow cytometry assay. Annexin V-FITC Apoptosis Detection kit (BD Pharmingen, Franklin Lakes, NJ, USA) was used to detect the apoptosis of cells. In brief, both floating and adherent cells were collected. After being washed and resuspended with PBS twice, the cells were stained with $3 \mu$ l Annexin V-FITC and $5 \mu \mathrm{l}$ PI for $20 \mathrm{~min}$ in the dark at room temperature. Then $150 \mu \mathrm{l}$ binding buffer was added to each sample of cells, after which the cells were analyzed by flow cytometry.

RNA interference. In order to reduce the expression of Atg7 and IRE1- $\alpha$ in LX-2 cells, small interfering RNAs (siRNAs) targeted against human Atg7 and IRE1- $\alpha$ were synthesized by GenePharma (Shanghai, China).

The sequence of Atg7-siRNA was 5'-GCCGUGGAAUUGA UGGUAUTT-3'. The sequence of IRE1- $\alpha$ was 5'-CTACTG GATGATAAATTTGCTTCA-3'. The negative control siRNA sequence was 5'-UUCUUCGAACGUGUCACGUTT-3'. Then LX-2 cells were transfected with Atg7-siRNA, IRE1- $\alpha$-siRNA or NC-siRNA, respectively, using Lipofectamine 2000 (Thermo Fisher Scientific, Inc., Waltham, MA, USA) according to the production's instruction manual.

Cell viability assay. The effects of caffeine on the viability of LX-2 cells were evaluated by Cell Counting Kit-8 (CCK-8) assay (Dojindo Laboratories, Kumamoto, Japan), as previously described in another article (21).

Intracellular $\mathrm{Ca}^{2+}$ detection. The levels of intracellular $\mathrm{Ca}^{2+}$ were detected using Fluo-3 AM (Beyotime Institute of Biotechnology, Shanghai, China). In brief, LX-2 cells were treated with different concentrations of caffeine $(0,10$ and $30 \mathrm{mM}$ ) for $24 \mathrm{~h}$, and the cells were harvested and washed twice using $\mathrm{Ca}^{2+}$-free PBS. Subsequently, the cells were resuspended in $5 \mu \mathrm{m}$ Fluo-3 AM for $30 \mathrm{~min}$ in the dark at $37^{\circ} \mathrm{C}$, after which the cells were washed twice using $\mathrm{Ca}^{2+}$-free PBS. To ensure that the Fluo-3 AM was completely transformed into Fluo-3 within the cells and to enable the Fluo-3 to more closely combine with $\mathrm{Ca}^{2+}$, the cells were placed quietly for $30 \mathrm{~min}$ in the dark at room temperature. Finally, the fluorescence intensities of Fluo-3 combined with $\mathrm{Ca}^{2+}$ were detected by flow cytometery.

Electron microscopy. Transmission electron microscopy was used to identify the ultrastructure of autophagosomes/autolysosomes as previously described elsewhere (22).

Statistical analysis. All data are presented as means \pm SD from at least triplicate parallel experiments. The Student's t-test was used to analyze the differences between two groups by SPSS version 23.0 (SPSS, Inc., Chicago, IL, USA). P<0.05 was considered as statistically significant.

\section{Results}

Caffeine inhibits the viability of LX-2 cells and induces cell apoptosis. To evaluate the direct effects of caffeine on HSCs in vitro, the LX-2 cell line, a type of immortal human HSCs, was applied in our study. After incubation with various concentrations of caffeine $(0,2.5,5,10,20$ and $30 \mathrm{mM})$ for $48 \mathrm{~h}$ or $20 \mathrm{mM}$ caffeine for the indicated times $(0,6,12,24,36$ and $48 \mathrm{~h}$ ), western blotting was used to detect the expression of $\alpha$-smooth muscle actin ( $\alpha$-SMA), a marker of activated HSCs. We found that the level of $\alpha$-SMA was inversely proportional to the caffeine concentration and to the duration of exposure. Particularly, caffeine decreased the $\alpha$-SMA/GAPDH ratio at 
A
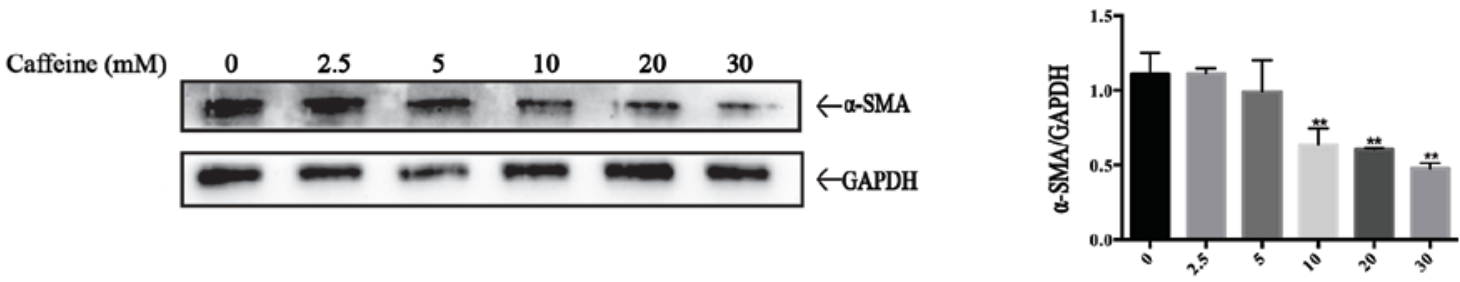

Concentration of caffeine (mM)

B
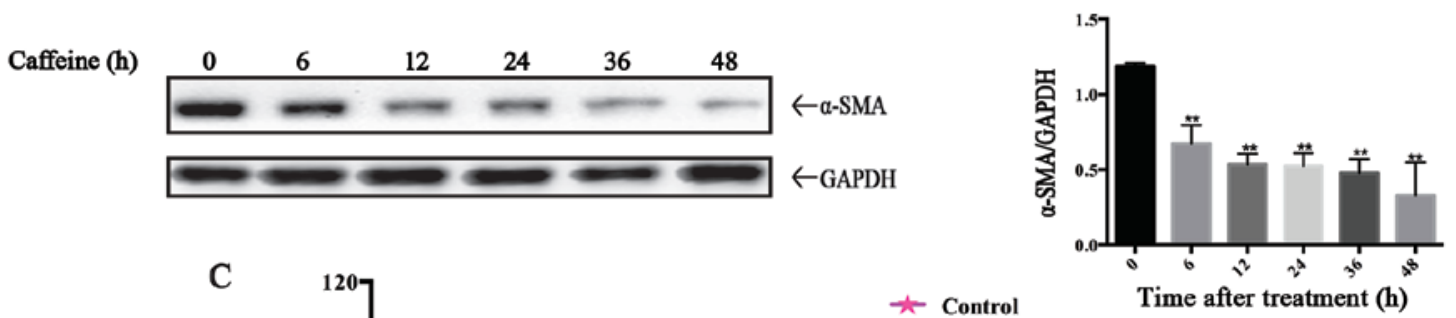

C
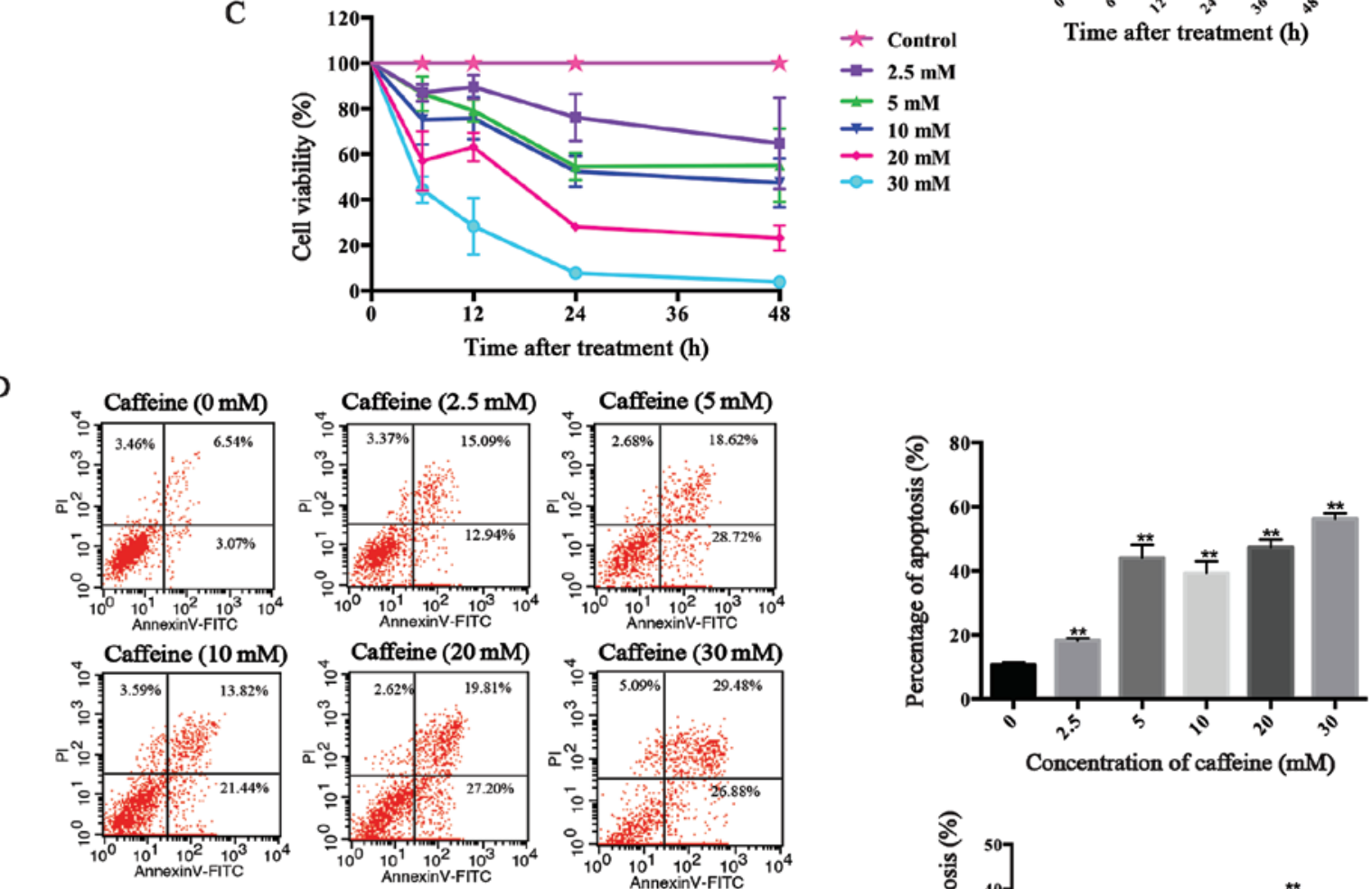

E
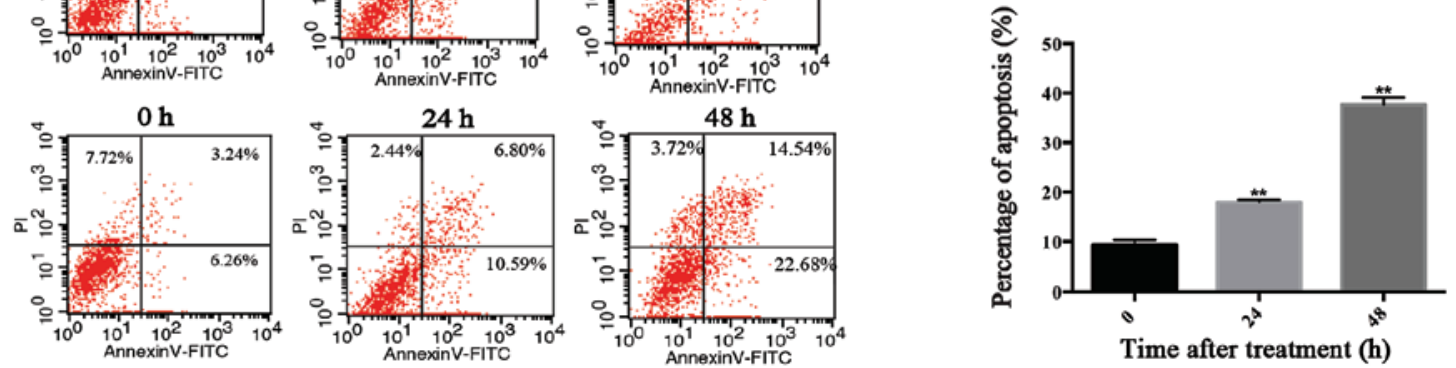

Figure 1. Caffeine inhibits the cell viability of LX-2 cells. (A and B) The expression of $\alpha$-smooth muscle actin $(\alpha-$ SMA) was analyzed by western blotting in LX-2 cells exposed to various concentrations of caffeine for $48 \mathrm{~h}$ (A) or (B) $20 \mathrm{mM}$ caffeine for the indicated times. Densitometric analysis of $\alpha$-SMA levels relative to GAPDH is shown for three respectively individual experiments. ${ }^{* *} \mathrm{P}<0.01$. (C) Impacts of caffeine on the viability of LX-2 cells by CCK-8 assay. (D) LX-2 cells were treated with $0,2.5,5,10,20$ and $30 \mathrm{mM}$ caffeine for $48 \mathrm{~h}$ or (E) $20 \mathrm{mM}$ caffeine for 0,24 and $48 \mathrm{~h}$, and the apoptosis of the cells was assessed by flow cytometry (the lower right region represents early apoptotic cells, the upper right region represents late apoptosis cells). Bars are the total percentages of early and late apoptotic cells which represent the means \pm SD of triplicate experiments. ${ }^{* *} \mathrm{P}<0.01$.

concentrations as low as $5 \mathrm{mM}$ (Fig. 1A) and at times as early as $6 \mathrm{~h}$ (Fig. 1B) in LX-2 cells. Furthermore, the results of the CCK-8 assay showed that the cell viability of the LX-2 cells was markedly reduced in a dose-dependent and time-dependent manner after treatment with caffeine (Fig. 1C). To investigate whether the caffeine-inhibited viability of LX-2 cells is associated with apoptosis, we examined the effects of caffeine on the apoptotic activity of cells by flow cytometry. The percentage of apoptotic cells which is the sum of early apoptotic cells (the low right region) and late apoptotic cells (the upper right region) was significantly increased in both a dose-dependent (Fig. 1D) and time-dependent manner (Fig. 1E) by incubation with caffeine.

Caffeine induces ER stress in the LX-2 cell line. Here we further investigated whether caffeine-induced HSC apoptosis is mediated by increasing ER stress. From the images 
A
a

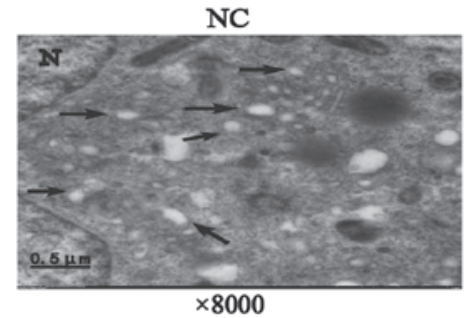

b

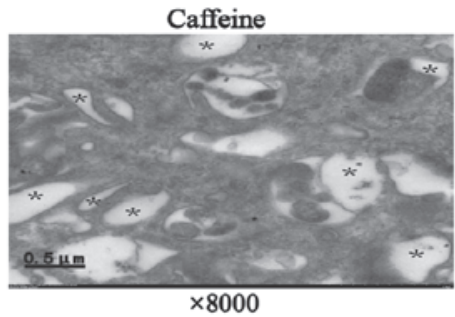

B
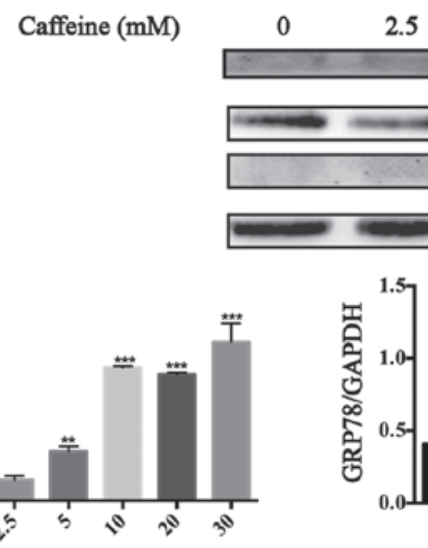
5

$10 \quad 20$

30

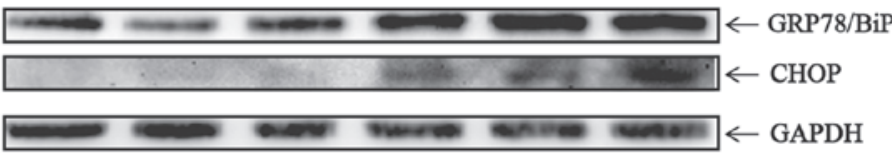

$\mathrm{C}$
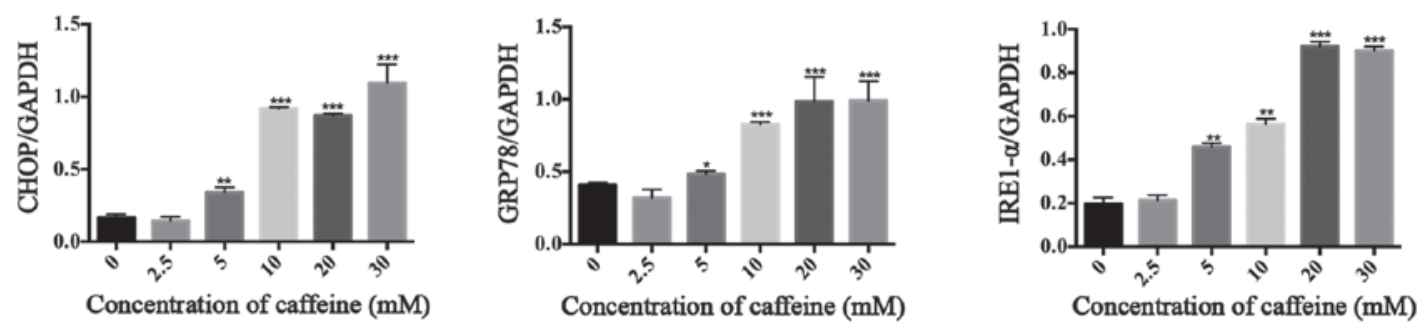

D Caffeine (h)
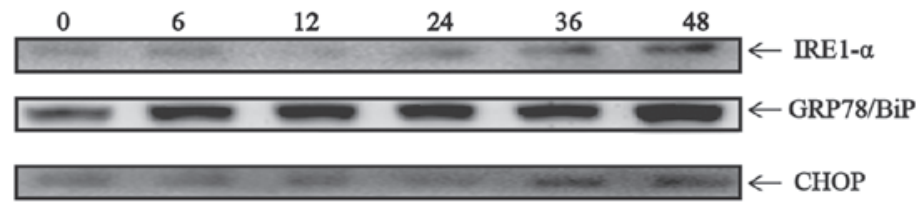

$\mathrm{E}$
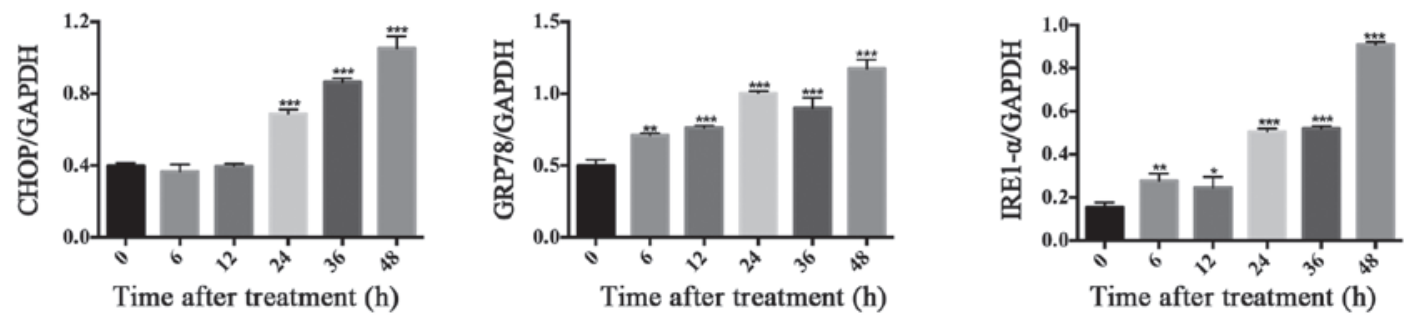

F
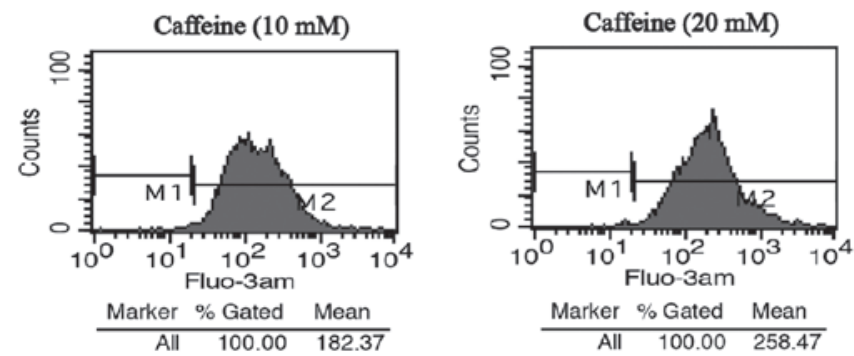

Figure 2. Endoplasmic reticulum (ER) stress induced by caffeine in the LX-2 cell line. (A) Electron microscopy (x8,000) shows dilated ER cavities in LX-2 cells in the presence of caffeine $(20 \mathrm{mM} / 48 \mathrm{~h}$ ). (a) Control cells and (b) caffeine-incubated cells are shown. $\rightarrow$, indicates normal ER; $\star$, represents dilated ER cavity; N, indicates the nucleus. (B) Western blotting and (C) densitometric analysis show a dose response of inositol requiring enzyme 1 (IRE1)- $\alpha$, GRP78/BiP, and CHOP accumulation in LX-2 cells incubated with various concentrations of caffeine $(0,2.5,5,10,20$ and $30 \mathrm{mM})$ for $48 \mathrm{~h}$. Bars represent the means \pm SD of three independent experiments. ${ }^{* * *} \mathrm{P}<0.01,{ }^{* * *} \mathrm{P}<0.001$. (D) Western blotting and (E) densitometric analysis show time-dependent changes in the expression of IRE1- $\alpha$, GRP78/BiP and CHOP in LX-2 cells incubated with $20 \mathrm{mM}$ caffeine for the indicated times. Bars represent the means \pm SD of three independent experiments. ${ }^{* *} \mathrm{P}<0.01,{ }^{* * *} \mathrm{P}<0.001$. (F) LX-2 cells were treated with various concentrations of caffeine $(0,10,30 \mathrm{mM})$ for $48 \mathrm{~h}$, after which cells were loaded with Fluo-3 AM for 30 min. Flow cytometry was used to detect the changes in cytosolic calcium in LX-2 cells.

taken by transmission electron microscopy, we identified many dilated cytoplasmic vacuoles in the LX-2 cells after caffeine $(20 \mathrm{mM})$ treatment for $48 \mathrm{~h}$. However, there were few dilated cytoplasmic vacuoles in the LX-2 cells cultured with DMEM not containing caffeine (Fig. 2A). These dilated cytoplasmic vacuoles can be recognized as dilated ER lumens as previously described (23), which indicates an increase in ER stress. Furthermore, we detected the expression of GRP78/BiP, IRE1- $\alpha$ and CHOP which all are markers of UPR. Notably, the ratio of GRP78/GAPDH, IRE1- $\alpha /$ 
A

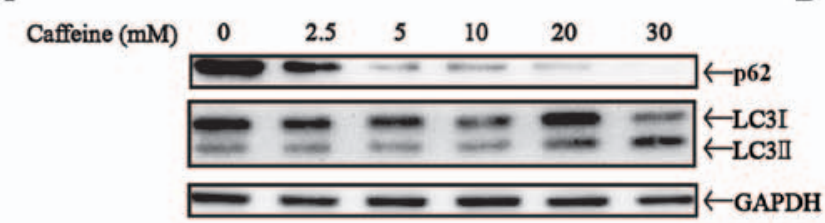

C

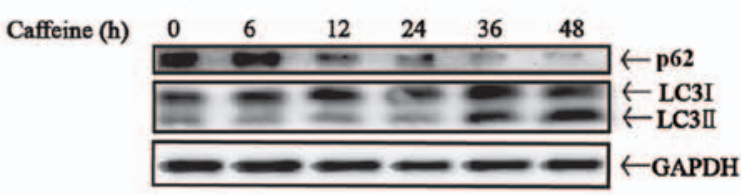

B

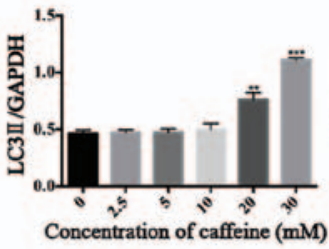

D

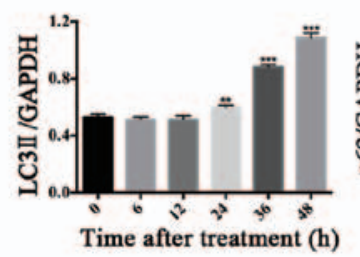

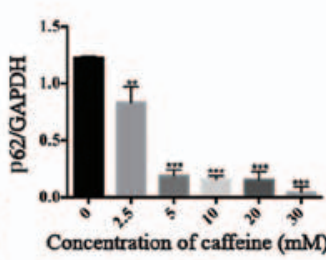

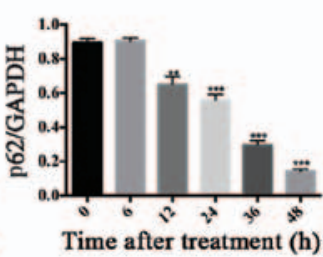

E
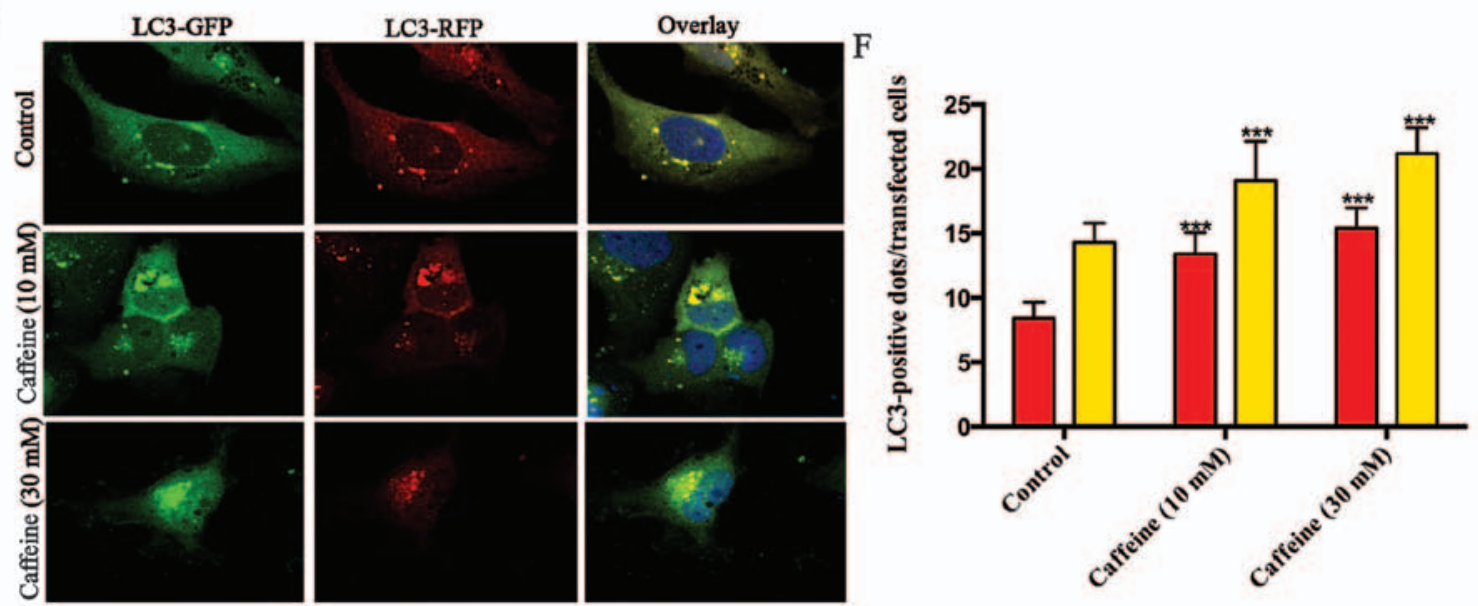

G a

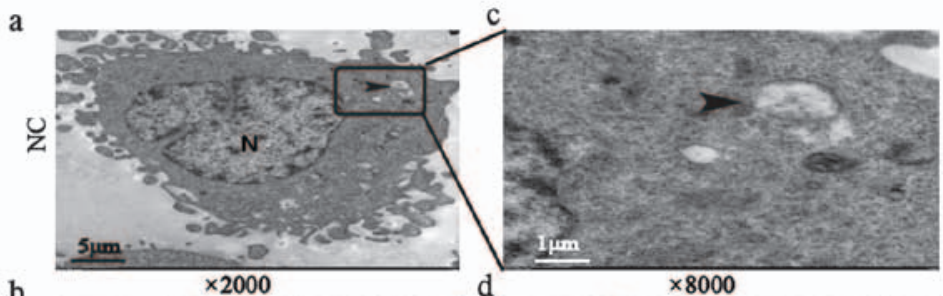

b

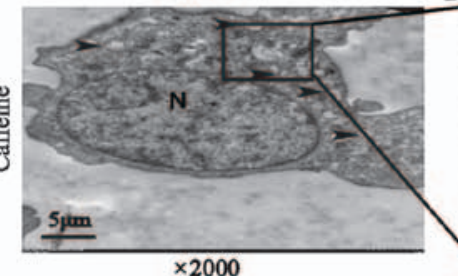

$\times 8000$

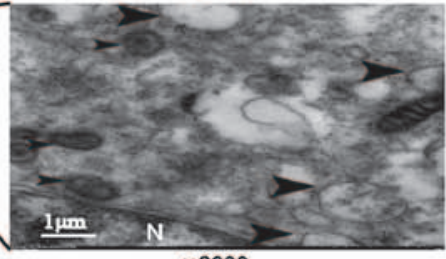

$\times 8000$

Figure 3. Autophagy flux is stimulated by caffeine in the LX-2 cell line. (A) Western blotting and (B) densitometric analysis show the dose response of p62 and LC3II accumulation in LX-2 cells incubated with various concentrations of caffeine $(0,2.5,5,10,20$ and $30 \mathrm{mM})$ for $48 \mathrm{~h}$. Bars represent the means \pm SD of three independent experiments. ${ }^{* *} \mathrm{P}<0.01,{ }^{* * *} \mathrm{P}<0.001$. (C) Western blotting and (D) densitometric analysis show time-dependent changes in the expression of p62 and LC3II in LX-2 cells incubated with $20 \mathrm{mM}$ caffeine for the indicated times. Bars represent the means $\pm \mathrm{SD}$ of three independent experiments. ${ }^{* *} \mathrm{P}<0.01$, ${ }^{* * * *} \mathrm{P}<0.001$. (E and F) Representative images and quantification of early-autophagosomes (yellow dots generated from overlapping GFP and RFP puncta) shown as yellow bars and late autolysosomes (red dots generated from RFP puncta) shown as red bars after $48 \mathrm{~h}$ of 10 and $30 \mathrm{mM}$ caffeine treatment vs. the controls (treated with $0 \mathrm{mM}$ caffeine) in LX-2 cells transfected with the RFP-GFP-hLC3 lentivirus. Error bar, standard deviation ( $\mathrm{n}=30$, from a total of independent three experiments), ${ }^{* * *} \mathrm{P}<0.001$. (G) Electron microscopy shows increased autophagosomes/autolysosomes in LX-2 cells incubated with $20 \mathrm{mM}$ caffeine for $48 \mathrm{~h}(\mathrm{~b}$ and d) vs. the control cells (a and c). Arrows indicate autophagosome or autolysosome structures; $\mathrm{N}$, indicates the nucleus. Magnification (x2,000 and $\mathrm{x} 8,000)$.

GAPDH and CHOP/GAPDH were parallel to the increase in caffeine concentration (Fig. 2B and C) and the duration of exposure (Fig. 2D and E). In addition, Fluo-3 AM was used to label cytoplasmic calcium in the LX-2 cells since calcium disturbance is another agent that induces ER stress. The results showed that caffeine significantly elevated the levels of cytoplasmic calcium in the LX-2 cells (Fig. 2F). Collectively, all these data demonstrated that caffeine induced ER stress in LX-2 cells.

Autophagic flux in the LX-2 cell line is increased after caffeine treatment. Four different methods were applied to 
A

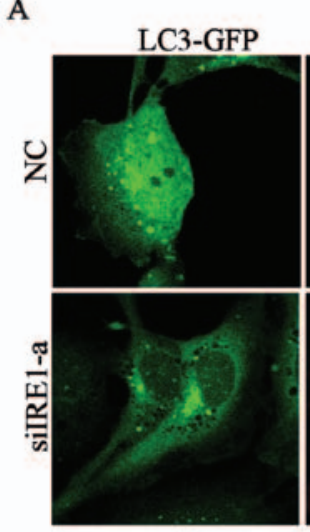

c

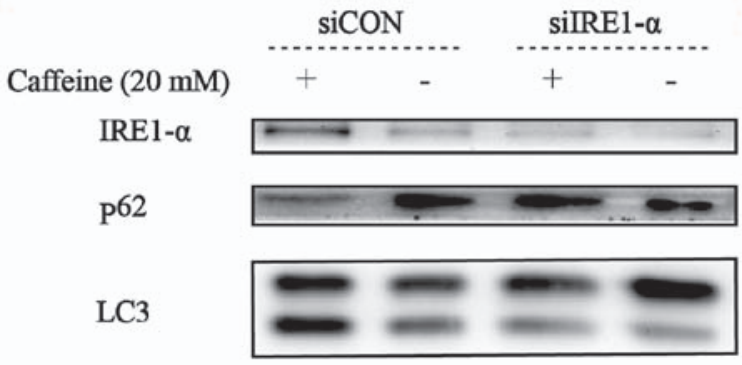

GAPDH
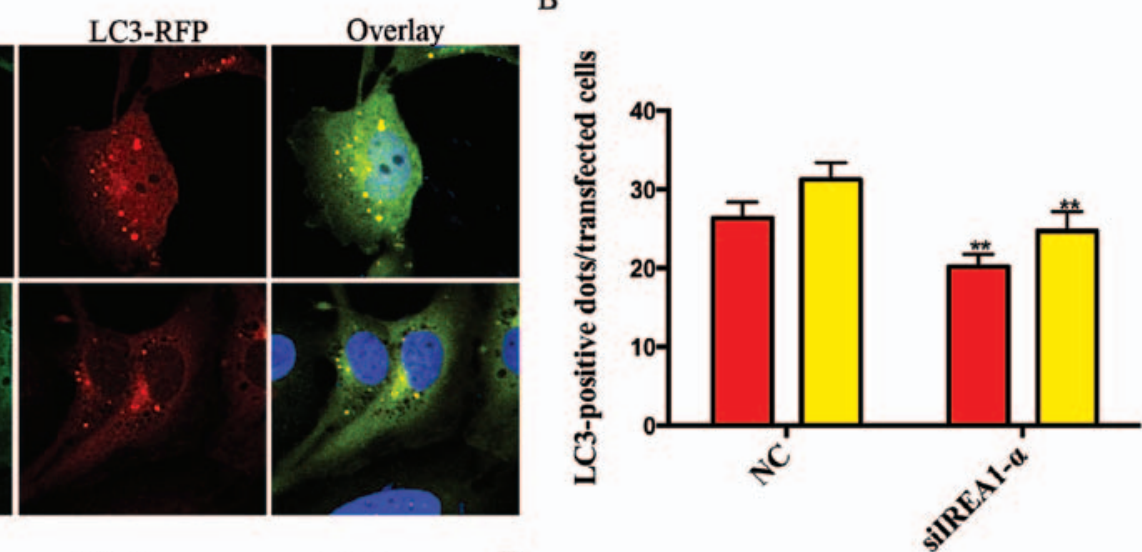

D

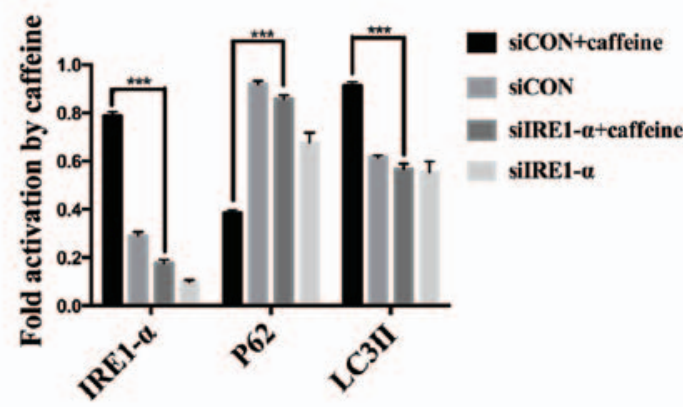

Figure 4. Autophagy in the LX-2 cell line treated with caffeine was stimulated by endoplasmic reticulum (ER) stress through the inositol-requiring enzyme 1 (IRE1)- $\alpha$ pathway. (A and B) Representative images and quantification of early autophagosomes (yellow dots generated from overlapping GFP and RFP puncta) shown as yellow bars and late autolysosomes (red dots generated from RFP puncta) shown as red bars after $48 \mathrm{~h}$ of $20 \mathrm{mM}$ caffeine treatment in LX-2 cells co-transfected with the RFP-GFP-hLC3 lentivirus and IRE1- $\alpha$-siRNAs vs. the control cells co-transfected with the RFP-GFP-hLC3 lentivirus and negative siRNAs. Error bar, standard deviation ( $\mathrm{n}=30$, from a total of independent three experiments), ${ }^{* *} \mathrm{P}<0.001$. (C) The expression of IRE1- $\alpha$, p62 and LC3 in LX-2 cells transfected with IRE1- $\alpha$-siRNAs or cells transfected with negative siRNAs after treatment with $0 / 20 \mathrm{mM}$ caffeine for $48 \mathrm{~h}$. (D) Densitometric analysis of IRE1- $\alpha$, p62 and LC3II levels relative to GAPDH is representative of three parallel experiments. ${ }^{* * *} \mathrm{P}<0.001$.

explore autophagic flux. Firstly, we detected the expression of LC3II, a good indicator for autophagosome formation, in LX-2 cells after caffeine treatment. Our results showed that the expression of LC3II was parallel to the increase in caffeine concentration (Fig. 3A and B) or the duration of exposure to caffeine (Fig. 3C and D). Secondly, we measured the accumulation of p62.p62 is a marker of autophagic flux whose level increases when autophagy is inhibited and decreases when autophagic flux is triggered. Interestingly, we found that caffeine-incubated LX-2 cells exhibited significant reduction in the level of p62 in a dose- and time-dependent manner. The results indicated that autophagy in LX-2 cells was induced by caffeine. Thirdly, autophagy is a dynamic process. The increase in LC3II level can be due to an increased autophagy presence or a result of the accumulation of autophagosomes caused by late autophagy inhibition. For the purpose of distinguishing autophagy induction from autophagy inhibition, a type of lentivirus encoding the RFP-GFP-LC3 fusion gene was used to our study. In this study, the autophagosomes are labeled with yellow dots (the overlay of green and red fluorescence), while autolysosomes are labeled with red dots only. If autophagic flux is upregulated, yellow and red dots are both increased, while only yellow dots are increased if the process of autophagosomes fusing with lysosomes is inhibited. It is the different numbers of red dots that represent changes in autophagic flux. To evaluate whether autophagic flux is upregulated by caffeine in LX-2 cells, we incubated
GFP-RFP-hLC3 lentivirus-transfected LX-2 cells with various concentrations of caffeine $(0,10$ and $30 \mathrm{mM})$. At $48 \mathrm{~h}$ points, fluorescence images were captured and quantification of the number of yellow and red dots was carried out. As shown in the fluorescence images, the number of yellow dots and red dots alone were both increased (Fig. 3E and F). Finally, electron microscopic evaluation showed an increased amount of autophagosomes/autolysosomes in the cells treated with caffeine (Fig. 3G). Taken together, these findings strongly demonstrated that autophagic flux in LX-2 cells was induced by caffeine.

The IRE1- $\alpha$ signaling pathway is required for activation of caffeine-induced autophagy in the LX-2 cell line. It has been reported that autophagy can be triggered by ER stress via the IRE1- $\alpha$ signaling pathway $(19,24)$. Herein, we found that caffeine contributed to autophagy and ER stress, as well as increased the expression levels of IRE1- $\alpha$ and CHOP in LX-2 cells. These findings prompted us to explore whether UPR acts as an upstream event of autophagy and the crucial role of IRE1- $\alpha$ in caffeine-treated LX-2 cells. To confirm the hypothesis, specific siRNAs directly against IRE1- $\alpha$ were used in the RFP-GFP-hLC3-transfected LX-2 cells. In these co-transfected cells, we observed that the numbers of yellow or red dots alone in the negative siRNA-transfected cells were both higher than those in the IRE1- $\alpha$ siRNA-transfected cells (Fig. 4A and B). We also found that the level of p62 

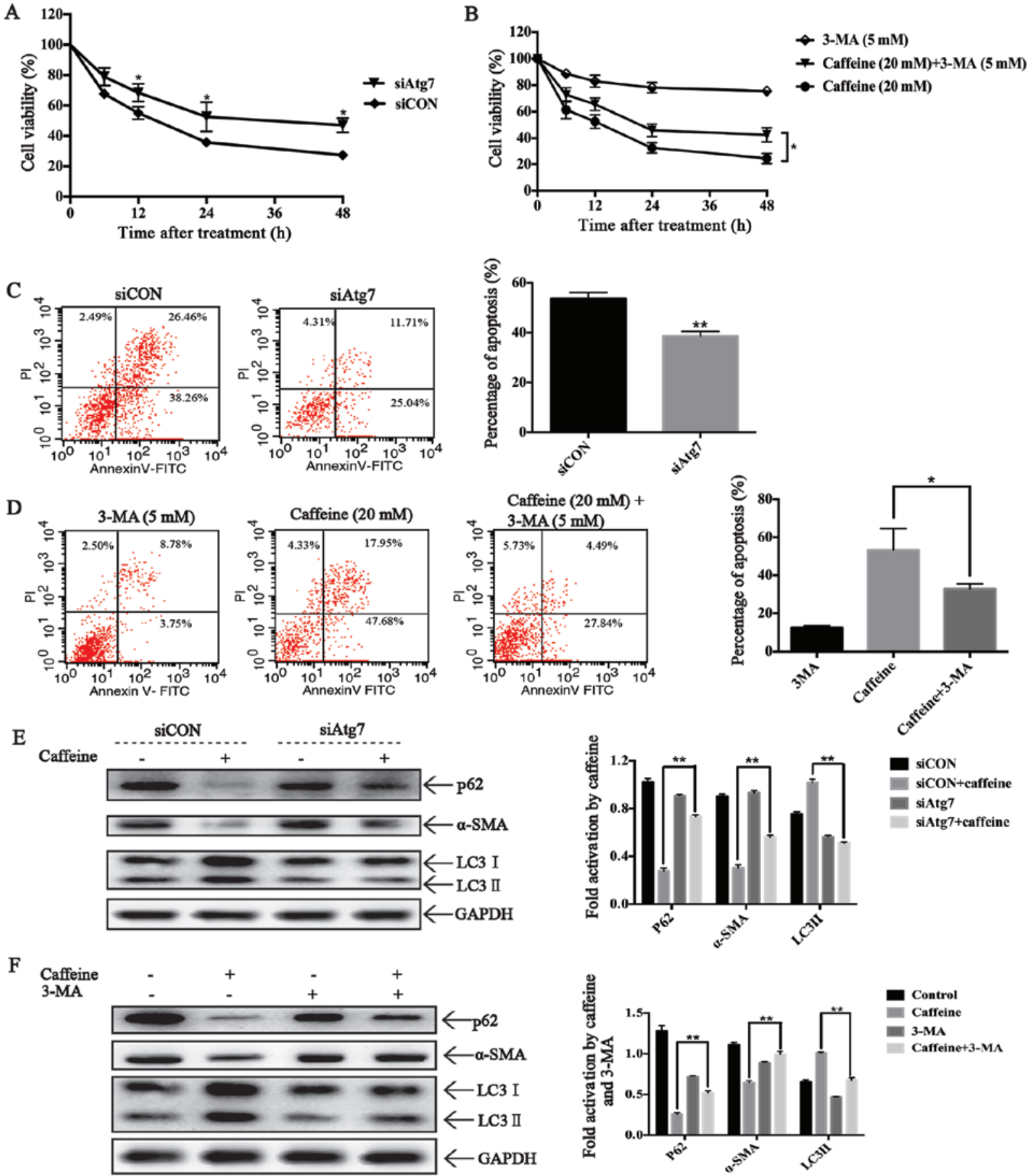

Figure 5. Effects of autophagy in LX-2 cells incubated with caffeine. (A) The viability of LX-2 cells transfected with Atg7-siRNAs or the cells transfected with negative siRNAs after $20 \mathrm{mM}$ caffeine treatment at the indicated times was detected by CCK-8 assay. (B) The viability of LX-2 cells treated with 3-MA $(5 \mathrm{mM})$, caffeine $(20 \mathrm{mM})$ and caffeine $(20 \mathrm{mM})$ together with 3-MA $(5 \mathrm{mM})$ was detected by CCK-8 assay. (C) The percentage of apoptosis of LX-2 cells transfected with Atg7-siRNAs or the cells transfected with negative siRNAs after $20 \mathrm{mM}$ caffeine treatment for $48 \mathrm{~h}$ was detected by flow cytometry. Bars represent the means $\pm \mathrm{SD}$ of triplicate experiments. ${ }^{* *} \mathrm{P}<0.01$. (D) The percentage of apoptotic LX-2 cells treated with $3-\mathrm{MA}(5 \mathrm{mM})$, caffeine $(20 \mathrm{mM})$ and caffeine $(20 \mathrm{mM})$ together with $3-\mathrm{MA}(5 \mathrm{mM})$ was detected by flow cytometry. Bars represent the means $\pm \mathrm{SD}$ of triplicate experiments. $\mathrm{P}<0.05$. (E) Western blotting and densitometric analysis show the expression of p62, $\alpha$-SMA and LC3II in LX-2 cells transfected with Atg7-siRNAs or the cells transfected with negative siRNAs after treatment with $0 / 20 \mathrm{mM}$ caffeine for $48 \mathrm{~h}$. Bars represent the means $\pm \mathrm{SD}$ of three independent experiments. ${ }^{* * *} \mathrm{P}<0.01$. (F) Western blotting and densitometric analysis show the expression of p62, $\alpha$-SMA and LC3II in LX-2 cells treated with 3-MA (5 mM), caffeine (20 mM), caffeine (20 mM) together with 3-MA $(5 \mathrm{mM})$ and none. Bars represent the means $\pm \mathrm{SD}$ of three independent experiments. ${ }^{* *} \mathrm{P}<0.01$.

accumulation in the IRE1- $\alpha$ siRNA-transfected cells was higher than that in the negative siRNA-transfected cells. Similarly, the accumulation of LC3II was decreased in the IRE1- $\alpha$ siRNA-transfected cells compared with the negative siRNAtransfected cells after caffeine treatment (Fig. 4C and D). All these data indicated that the IRE1- $\alpha$ pathway was an upstream event of caffeine-induced autophagy in the LX-2 cell line.

Autophagy triggered by caffeine inhibits cell viability, induces apoptosis and suppressesactivation of $L X-2$ cells. To investigate 
the roles of autophagy on cell viability in caffeine-treated LX-2 cells, we used Atg7-siRNAs to block autophagosome formation and 3-MA to inhibit the activation of autophagic flux in LX-2 cells. As expected, Atg7-knockdown reduced LC3II accumulation and increased the expression level of p62, which significantly increased the cell viability of the LX-2 cells after caffeine treatment (Fig. 5A). These findings strongly suggest the involvement of autophagy in caffeine-inhibited viability of LX-2 cells. Furthermore, Atg7-knockdown also strongly decreased the extent of apoptotic death of the LX-2 cells after caffeine incubation (Fig. 5C), suggesting that autophagy is essential for caffeine-induced apoptosis of LX-2 cells. Additionally, Atg7-knockdown rescued the expression of $\alpha$-SMA, indicating a direct link between autophagy and LX-2 activation. Similar results were also found in 3-MA and caffeine co-treated LX-2 cells (Fig. 5B, D and F). All these results indicated that autophagy induced apoptosis, inhibited cell viability and suppressed cell activation in caffeine-treated LX-2 cells.

\section{Discussion}

Recently, many studies have demonstrated that the long-term consumption of caffeine is associated with lower risks for chronic liver diseases, such as nonalcoholic fatty disease and liver fibrosis (25-27). Moreover, other studies have reported that caffeine attenuates liver fibrosis by directly inducing apoptosis of HSCs (18). However, a better understanding of the pathways implicated in this process remains to be characterized. In the present study, our findings established autophagy mediated by ER stress as a novel pathway implicated in caffeine-induced HSC apoptosis.

To the best of our knowledge, liver fibrosis is triggered by hepatocyte apoptosis, while induction of activated HSC apoptosis can reverse the progression of liver fibrosis (28-30). Importantly, our study showed that caffeine markedly reduced the cell viability of activated HSCs and downregulated the expression of $\alpha$-SMA in vitro, suggesting caffeine as a suppressor of HSC activation. Furthermore, in agreement with other studies, we established the role of caffeine as an important driver of stellate cell apoptosis. The results above correspond to the principle that augmentation of HSC apoptosis is a potential approach through which to promote the resolution of liver fibrosis (31), therefore, epitomizing an anti-fibrotic function of caffeine.

Morphological analysis found that caffeine induced an increase in vacuoles which were recognized as enlarged ERs in HSCs. One reasonable explanation for these enlarged vacuoles is induction of the UPR, a self-protective process initially occurring after ER stress $(22,24)$. Moreover, treatment with caffeine caused upregulated expression of GRP78/BiP. GRP78/BiP is an ER chaperone that normally binds with three transmembrane proteins, IRE1- $\alpha$, activating transcription factor (ATF)-6 $\alpha$ and RNA-activated protein kinase-like ER kinase (PERK) $(32,33)$. Upon ER stress, GRP78/BiP dissociates with the three transmembrane proteins described above, causing the activation of PERK and IRE1- $\alpha$ by trans-autophosphorylation as well as ATF6 $\alpha$ via proteolytic processing $(34,35)$. The IRE1- $\alpha /$ CHOP pathway is the most conserved branch of the UPR among the three (7). Notably, our study showed that the accumulation of IRE1- $\alpha$ and CHOP in HSCs was rapidly increased after caffeine treatment. Collectively, these events strongly demonstrated that the ER stress-dependent UPR signaling pathway is triggered in caffeine-treated HSCs.

Although the UPR is an adaptive process that compensates for the damage caused by ER stress, prolonged and massive ER stress that cannot be alleviated by UPR will induce cell apoptosis $(12,36)$. In the present study, we revealed that caffeine inhibited the cell viability of HSCs by inducing apoptosis; meanwhile, ER stress was stimulated after caffeine treatment in HSCs. These results can be explained by the principle that treatment with caffeine induces prolonged and sustained ER stress in HSCs and the ER stress-related damage cannot be eliminated by UPR, eventually, resulting in apoptotic death $(22,37)$. Additionally, we also found that the level of cytosolic calcium was increased after caffeine incubation in HSCs. To date, perturbations in ER calcium have been demonstrated to have a close associate with apoptosis effectors (38). In certain cell lines, ER stress inducers promote calcium to be released from the ER accompanied by some related apoptosis effectors released from mitochondria $(33,39)$. Although the exact functional role of calcium mobilization in caffeine-mediated ER stress remains unclear, the possibility that the calcium released from the ER triggers ER stress and apoptosis seems reasonable.

Recent research of the mechanisms by which caffeine ameliorates the severity of NAFLD has revealed that caffeine markedly decreased intracellular lipids via upregulating autophagic flux in hepatocytes (27). Analogously, in the present research, we demonstrated that the biochemical hallmarks of autophagic flux in HSCs were induced, for example, LC3II accumulation and GFP-RFP-LC3 redistribution in early and late autophagosomes. Furthermore, many doublemembrane vacuolar structures, the morphological pattern of autophagosomes, were found in caffeine-treated HSCs, while few were observed in the controls. Notably, we also showed that GFP-RFP-LC3 redistribution in early and late autophagosomes and LC3II accumulation were rapidly decreased after knockdown of IRE1- $\alpha$. In addition, accumulation of p62 was significantly compromised in the IRE1- $\alpha$ siRNA-transfected HSCs. These data not only demonstrated that caffeine induced the activation of autophagy in HSCs, but also established the role of ER stress as an important driver of autophagic flux induced by caffeine in HSCs.

The primary role of autophagy in cells is complicated, sometimes paradoxical. Whether autophagy protects cells from death or promotes cell death depends on the cellular context, the duration and strength of stimuli $(40,41)$. Previously, one study revealed that caffeine induced apoptosis in SH-SY5Y, PC12D and HeLa cells by enhancement of autophagy (19). Similarly, the data presented here showed that both of the genetic and chemical autophagy inhibitors, Atg7-siRNAs and 3-MA, alleviated caffeine-induced apoptosis. Coincident with the alleviation of cell death by autophagy, the cell viability was increased in the Atg7-knockdown and 3-MA-pretreated HSCs after caffeine treatment. These data strongly demonstrate that autophagy induced by caffeine promoted apoptotic cell death rather than cell survival in the HSCs. To date, many studies have reported that autophagy removes superabundant proteins 
when the accumulation of unfolded or misfolded proteins surpasses the ability of the degradation system mediated by the proteasome $(42,43)$. The protective function of autophagy was contradictory with our results. One plausible explanation is that the outcome of ER stress-mediated autophagy depends on the type of stimulants and the cellular context. Notably, our findings rightly identify with the anti-fibrosis mechanism of caffeine. In brief, caffeine suppresses activation of HSCs by induction of autophagy via the IRE1- $\alpha$ pathway. And once the activated HSCs are eliminated, the progression of liver fibrosis can be attenuated and reversed. Viewed this way, our finding is consistent with previous studies on the induction of apoptotic cell death by caffeine and provides novel insight into the mechanism involved in the anti-fibrotic function of caffeine.

Additionally, it is worth noting that in our study the concentrations of caffeine that induced apoptosis of HSCs were in a high range. The results disclosed that only a high concentration of caffeine attenuated liver fibrosis, which is in accordance with previous research that individuals who consume a large amount of caffeine every day have the lowest risk of liver disease (44).

In conclusion, our data highlight the importance of autophagy mediated by ER stress in caffeine-treated HSCs as a main driver of apoptosis, extending the mechanisms of the attenuation of liver fibrosis by caffeine. We speculate that moderate to high caffeine intake can be useful in the treatment of liver fibrosis.

\section{Acknowledgements}

This study was supported by the National Natural Science Foundation of China (no. 81070358), (http://www.nsfc.gov. cn) to JQY.

\section{References}

1. Tsochatzis EA, Bosch $\mathrm{J}$ and Burroughs AK: Liver cirrhosis. Lancet 383: 1749-1761, 2014.

2. Friedman SL: Hepatic stellate cells: protean, multifunctional, and enigmatic cells of the liver. Physiol Rev 88: 125-172, 2008.

3. Mederacke I, Hsu CC, Troeger JS, Huebener P, Mu X, Dapito DH, Pradere JP and Schwabe RF: Fate tracing reveals hepatic stellate cells as dominant contributors to liver fibrosis independent of its aetiology. Nat Commun 4: 2823, 2013.

4. Thoen LF, Guimarães EL, Dollé L, Mannaerts I, Najimi M, Sokal E and van Grunsven LA: A role for autophagy during hepatic stellate cell activation. J Hepatol 55: 1353-1360, 2011

5. Parola M, Marra F and Pinzani M: Myofibroblast-like cells and liver fibrogenesis: emerging concepts in a rapidly moving scenario. Mol Aspects Med 29: 58-66, 2008.

6. Glick D, Barth S and Macleod KF: Autophagy: cellular and molecular mechanisms. J Pathol 221: 3-12, 2010.

7. Hernández-Gea V, Hilscher M, Rozenfeld R, Lim MP, Nieto N, Werner S, Devi LA and Friedman SL: Endoplasmic reticulum stress induces fibrogenic activity in hepatic stellate cells through autophagy. J Hepatol 59: 98-104, 2013.

8. Ding WX and Yin XM: Sorting, recognition and activation of the misfolded protein degradation pathways through macroautophagy and the proteasome. Autophagy 4: 141-150, 2008.

9. Bernales S, Papa FR and Walter P: Intracellular signaling by the unfolded protein response. Annu Rev Cell Dev Biol 22: 487-508, 2006

10. Momoi T: Conformational diseases and ER stress-mediated cell death: apoptotic cell death and autophagic cell death. Curr Mol Med 6: 111-118, 2006.

11. Koo JH, Lee HJ, Kim W and Kim SG: Endoplasmic reticulum stress in hepatic stellate cells promotes liver fibrosis via PERK-mediated degradation of HNRNPA1 and up-regulation of SMAD2. Gastroenterology 150: 181-193.e8, 2016.
12. Ron D and Walter P: Signal integration in the endoplasmic reticulum unfolded protein response. Nat Rev Mol Cell Biol 8: 519-529, 2007.

13. Xu C, Bailly-Maitre B and Reed JC: Endoplasmic reticulum stress: cell life and death decisions. J Clin Invest 115: 2656-2664, 2005.

14. Meares GP, Mines MA, Beurel E, Eom TY, Song L, Zmijewska AA and Jope RS: Glycogen synthase kinase-3 regulates endoplasmic reticulum (ER) stress-induced CHOP expression in neuronal cells. Exp Cell Res 317: 1621-1628, 2011.

15. Choi AY, Choi JH, Yoon H, Hwang KY, Noh MH, Choe W, Yoon KS, Ha J, Yeo EJ and Kang I: Luteolin induces apoptosis through endoplasmic reticulum stress and mitochondrial dysfunction in Neuro-2a mouse neuroblastoma cells. Eur J Pharmacol 668: 115-126, 2011.

16. Hsu SJ, Lee FY, Wang SS, Hsin IF, Lin TY, Huang HC, Chang CC, Chuang CL, Ho HL, Lin HC, et al: Caffeine ameliorates hemodynamic derangements and portosystemic collaterals in cirrhotic rats. Hepatology 61: 1672-1684, 2015.

17. Gressner OA, Lahme B, Rehbein K, Siluschek M, Weiskirchen R and Gressner AM: Pharmacological application of caffeine inhibits TGF- $\beta$-stimulated connective tissue growth factor expression in hepatocytes via PPARgamma and SMAD2/3-dependent pathways. J Hepatol 49: 758-767, 2008.

18. Shim SG, Jun DW, Kim EK, Saeed WK, Lee KN, Lee HL, Lee OY, Choi HS and Yoon BC: Caffeine attenuates liver fibrosis via defective adhesion of hepatic stellate cells in cirrhotic model. J Gastroenterol Hepatol 28: 1877-1884, 2013.

19. Saiki S, Sasazawa Y, Imamichi Y, Kawajiri S, Fujimaki T, Tanida I, Kobayashi H, Sato F, Sato S, Ishikawa K, et al: Caffeine induces apoptosis by enhancement of autophagy via PI3K/Akt/mTOR/p70S6K inhibition. Autophagy 7: 176-187, 2011.

20. Yan X, Shan Z, Yan L, Zhu Q, Liu L, Xu B, Liu S, Jin Z and Gao Y: High expression of zinc-finger protein X-linked promotes tumor growth and predicts a poor outcome for stage II/III colorectal cancer patients. Oncotarget 7: 19680-19692, 2016.

21. Ou B, Zhao J, Guan S, Wangpu X, Zhu C, Zong Y, Ma J, Sun J, Zheng M, Feng H, et al: Plk2 promotes tumor growth and inhibits apoptosis by targeting Fbxw7/Cyclin E in colorectal cancer. Cancer Lett 380: 457-466, 2016.

22. Shi YH, Ding ZB, Zhou J, Hui B, Shi GM, Ke AW, Wang XY, Dai Z, Peng YF, Gu CY, et al: Targeting autophagy enhances sorafenib lethality for hepatocellular carcinoma via ER stress-related apoptosis. Autophagy 7: 1159-1172, 2011.

23. Ding WX, Ni HM, Gao W, Hou YF, Melan MA, Chen X, Stolz DB, Shao ZM and Yin XM: Differential effects of endoplasmic reticulum stress-induced autophagy on cell survival. J Biol Chem 282: 4702-4710, 2007.

24. Xu WH, Liu ZB, Hou YF, Hong Q, Hu DL and Shao ZM: Inhibition of autophagy enhances the cytotoxic effect of PA-MSHA in breast cancer. BMC Cancer 14: 273, 2014.

25. Friedrich K, Smit M, Wannhoff A, Rupp C, Scholl SG, Antoni C, Dollinger M, Neumann-Haefelin C, Stremmel W, Weiss KH, et al: Coffee consumption protects against progression in liver cirrhosis and increases long-term survival after liver transplantation. J Gastroenterol Hepatol 31: 1470-1475, 2016.

26. Wadhawan M and Anand AC: Coffee and liver disease. J Clin Exp Hepatol 6: 40-46, 2016.

27. Sinha RA, Farah BL, Singh BK, Siddique MM, Li Y, Wu Y, Ilkayeva OR, Gooding J, Ching J, Zhou J, et al: Caffeine stimulates hepatic lipid metabolism by the autophagy-lysosomal pathway in mice. Hepatology 59: 1366-1380, 2014.

28. Elsharkawy AM, Oakley F and Mann DA: The role and regulation of hepatic stellate cell apoptosis in reversal of liver fibrosis. Apoptosis 10: 927-939, 2005.

29. Jeschke MG, Gauglitz GG, Song J, Kulp GA, Finnerty CC, Cox RA, Barral JM, Herndon DN and Boehning D: Calcium and ER stress mediate hepatic apoptosis after burn injury. J Cell Mol Med 13 (8B): 1857-1865, 2009.

30. Ji C: Dissection of endoplasmic reticulum stress signaling in alcoholic and non-alcoholic liver injury. J Gastroenterol Hepatol 23 (Suppl 1): S16-S24, 2008.

31. Wang Y, Gao J, Zhang D, Zhang J, Ma J and Jiang H: New insights into the antifibrotic effects of sorafenib on hepatic stellate cells and liver fibrosis. J Hepatol 53: 132-144, 2010.

32. Shimada Y, Kobayashi H, Kawagoe S, Aoki K, Kaneshiro E, Shimizu H, Eto Y, Ida H and Ohashi T: Endoplasmic reticulum stress induces autophagy through activation of p38 MAPK in fibroblasts from Pompe disease patients carrying c.546G $>\mathrm{T}$ mutation. Mol Genet Metab 104: 566-573, 2011. 
33. Malhi $\mathrm{H}$ and Kaufman RJ: Endoplasmic reticulum stress in liver disease. J Hepatol 54: 795-809, 2011.

34. Bertolotti A, Zhang Y, Hendershot LM, Harding HP and Ron D: Dynamic interaction of $\mathrm{BiP}$ and ER stress transducers in the unfolded-protein response. Nat Cell Biol 2: 326-332, 2000.

35. Oikawa D, Kimata Y, Kohno K and Iwawaki T: Activation of mammalian IRElalpha upon ER stress depends on dissociation of $\mathrm{BiP}$ rather than on direct interaction with unfolded proteins. Exp Cell Res 315: 2496-2504, 2009.

36. Szegezdi E, Logue SE, Gorman AM and Samali A: Mediators of endoplasmic reticulum stress-induced apoptosis. EMBO Rep 7: 880-885, 2006.

37. Hwang MS and Baek WK: Glucosamine induces autophagic cell death through the stimulation of ER stress in human glioma cancer cells. Biochem Biophys Res Commun 399: 111-116, 2010

38. Deniaud A, Sharaf el dein O, Maillier E, Poncet D, Kroemer G, Lemaire $\mathrm{C}$ and Brenner $\mathrm{C}$ : Endoplasmic reticulum stress induces calcium-dependent permeability transition, mitochondrial outer membrane permeabilization and apoptosis. Oncogene 27: 285-299, 2008

39. Lawless MW, Mankan AK, White M, O'Dwyer MJ and Norris S: Expression of hereditary hemochromatosis C282Y HFE protein in HEK293 cells activates specific endoplasmic reticulum stress responses. BMC Cell Biol 8: 30, 2007.
40. Kondo Y, Kanzawa T, Sawaya R and Kondo S: The role of autophagy in cancer development and response to therapy. Nat Rev Cancer 5: 726-734, 2005.

41. Levine B and Kroemer G: Autophagy in the pathogenesis of disease. Cell 132: 27-42, 2008.

42. Criollo A, Maiuri MC, Tasdemir E, Vitale I, Fiebig AA, Andrews D, Molgó J, Díaz J, Lavandero S, Harper F, et al: Regulation of autophagy by the inositol trisphosphate receptor. Cell Death Differ 14: 1029-1039, 2007.

43. Levine B and Klionsky DJ: Development by self-digestion: molecular mechanisms and biological functions of autophagy. Dev Cell 6: 463-477, 2004.

44. Hu G, Tuomilehto J, Pukkala E, Hakulinen T, Antikainen R, Vartiainen E and Jousilahti P: Joint effects of coffee consumption and serum gamma-glutamyltransferase on the risk of liver cancer. Hepatology 48: 129-136, 2008. 